\title{
Avaliação de sintomas e consequências da tensão pré-menstrual em acadêmicas de uma universidade de Minas Gerais (Brasil)
}

\author{
Evaluation of symptoms and consequences from premenstrual tension in female \\ undergraduates at a university in Minas Gerais (Brazil)
}
Evaluación de síntomas y consecuencias de la tensión pre-menstrual en académicas de una universidad de Minas Gerais (Brasil)

Carolina da Cruz Lopes ${ }^{1}$, Luiz Henrique Torres Cota ${ }^{1}$, Luiz Henrique Marra da Silva Ribeiro ${ }^{1}$, Murilo Campos Silva ${ }^{1}$, Paula Miranda Esteves Orsi ${ }^{1}$, Andreia Majella da Silva Duarte Esteves ${ }^{1}$, Cláudio Daniel Cerdeira ${ }^{1 *}$, Gérsika Bitencourt Santos Barros ${ }^{1}$.

\section{RESUMO}

Objetivo: Verificar os grupos de sintomas e consequências da tensão pré-menstrual (TPM) em acadêmicas. Métodos: Entre fevereiro e março de 2017, através de questionários individuais, os sintomas e consequências da TPM foram levantados em uma amostra de 404 universitárias, com 18 a 45 anos, de diferentes cursos de graduação. Resultados: Todas as entrevistadas apresentaram algum tipo de sinal ou sintoma de TPM. Os grupos de maior incidência para os "sintomas físicos" foram: mamas inchadas e doloridas $(61,4 \%)$, desejo por alimentos $(59,9 \%)$, fome $(54,4 \%)$, dor de cabeça $(45,5 \%)$, inchaço em alguma parte do corpo $(38,1 \%)$, sensação de ganho de peso $(34,4 \%)$, aumento do desejo sexual $(31,9 \%)$, dor nas costas $(28,5 \%)$, e sensação de peso no abdome (25,2\%); "sintomas psicológicos": irritabilidade $(81,4 \%)$, impaciência $(62,6 \%)$, vontade de chorar $(60,8 \%)$, ansiedade $(55,2 \%)$ e tristeza $(50,9 \%)$; "consequências" (ambientes fora de casa): responde de forma hostil às pessoas $(53,2 \%)$, afronta mais as pessoas $(36,1 \%)$, quietude $(34,4 \%)$ e gera clima de tensão $(27,0 \%)$; e "consequências" (ambiente familiar): briga mais com familiares $(68,1 \%)$ e cria intrigas com/entre eles $(43,1 \%)$. Conclusão: Todas as estudantes apresentam alterações de comportamento no período de TPM, podendo isto influenciar em suas rotinas diárias.

Palavras chave: Tensão Pré-menstrual, Sintomas Físicos, Sintomas Psíquicos, Relação com Familiares, Relação com Comunidade e Instituições.

\begin{abstract}
Objective: To verify groups of symptoms and consequences from premenstrual tension (PMT) in female undergraduates. Methods: Between February and March 2017, through an individual questionnaire, symptoms and consequences caused by PMT were gathered from 404 female undergraduates, age range 18-45 years, from different undergraduate courses. Results: All of interviews presented some type of symptoms and/or consequences from PMT. The highest occurrence groups were: for "physical symptoms": swelling and sore breasts (61.4\%), food desire $(59.9 \%)$, hunger $(54.4 \%)$, headache $(45.5 \%)$, swelling in another part of the body (38.1\%), feeling of weight gain $(34.4 \%)$, increase in sexual desire $(31.9 \%)$, backache $(28.2 \%)$, and feeling of heavy abdomen (25.2\%); for "psychic symptoms": irritability (81.4\%), impatience $(62.6 \%)$, craving $(60.8 \%)$, anxiety $(55.2 \%)$, and sadness $(50.9 \%)$; for "consequences" (out-ofhome environments): responding in a hostile manner to people $(53.2 \%)$, distresses people more $(36.1 \%)$, "quietude" (34.4\%), and creates a tension at the environment (27.0\%); and for "consequences" (family environment): more troubles with family members (68.1\%) and intrigues with/between them (43.0\%). Conclusion: All of interviews exhibited symptoms with behavioral changes during the PMT period, which can influence in their daily routine.
\end{abstract}

Keywords: Premenstrual tension, Physic Symptoms, Psychic Symptoms, Family Relations, CommunityInstitutional Relations.

${ }^{1}$ Universidade José do Rosário Vellano - Unifenas/Alfenas, Alfenas, Minas Gerais (MG), Brasil.

*E-mail: daniel.cerdeira.84@gmail.com

SUBMETIDO EM: 6/2019

ACEITO EM: 7/2019

PUBLICADO EM: 8/2019 


\section{RESUMEN}

Objetivo: Verificar los grupos de síntomas y consecuencias de la tensión premenstrual (TPM) en académicas. Métodos: Entre febrero y marzo de 2017, a través de cuestionarios, los síntomas y consecuencias de TPM se levantaron entre 404 académicas, con 18-45 años, y provenientes de diferentes cursos de graduación. Resultados: Todas las entrevistadas fueron caracterizadas con algún tipo de señal o síntoma de TPM. Se identificaron grupos de mayor incidencia para los "síntomas físicos": mamas hinchadas y doloridas $(61,4 \%)$, deseo por alimentos $(59,9 \%)$, hambre $(54,4 \%)$, dolor de cabeza $(45,5 \%)$, hinchazón en alguna parte del cuerpo $(38,1 \%)$, sensación de aumento de peso $(34,4 \%)$, aumento del deseo sexual $(31,9 \%)$, dolor de espalda $(28,5 \%)$, y sensación de peso en el abdomen $(25,2 \%)$; "Síntomas psicológicos": irritabilidad $(81,4 \%)$, impaciencia $(62,6 \%)$, voluntad de llorar $(60,8 \%)$, ansiedad $(55,2 \%)$ y tristeza $(50,9 \%)$; "Consecuencias" (ambientes fuera de casa): responde de forma hostil a las personas $(53,2 \%)$, afronta más a las personas $(36,1 \%)$, quietud $(34,4 \%)$ y genera clima de tensión $(27,0) \%$; y "consecuencias" (ambiente familiar): pelea más con familiares $(68,1 \%$ ) y crea intrigas con/entre ellos $(43,1 \%)$. Conclusión: Todas las académicas presentaron alteraciones de comportamiento durante el período de la TPM, y estas alteraciones pueden influir en sus rutinas diarias.

Palabras clave: Tensión Pre-menstrual, Síntomas Físicos, Síntomas Psíquicos, Relación con Familiares, Relación con Comunidad e Instituciones.

\section{INTRODUÇÃO}

A tensão pré-menstrual (TPM) é caracterizada por um conjunto de sintomas físicos, emocionais e comportamentais que antecedem o primeiro dia da menstruação e tendem a aliviar-se com o início do fluxo, sendo um distúrbio altamente prevalente em mulheres na idade fértil (BRILHANTE AVM et al., 2010). Os sintomas da TPM podem ser divididos em 2 grupos: físicos e psíquicos (APPROBATO MS et al., 2001). Dentre os sintomas físicos mais comuns estão os estados congestivos que afetam principalmente as mamas, abdome e pelve, a retenção hídrica e outras manifestações como a enxaqueca, dentre outros. Dentre os sintomas psíquicos com maior frequência estão à incapacidade de concentração, labilidade afetiva, perturbações no sono, agressividade, irritabilidade, tensão nervosa, humor variável, depressão, ansiedade, crises de choro e desânimo (PAIVA PSC et al., 2010).

Não existe um fator único relacionado à etiologia da Síndrome da TPM. Sua natureza ainda não é especificamente conhecida, porém, um ou mais fatores responsáveis pela sintomatologia são produzidos pelo corpo lúteo e regridem após os níveis de progesterona e estrógeno alcançarem suas concentrações na fase folicular, no final da fase lútea (MURAMATSU $\mathrm{CH}$ et al., 2001). O diagnóstico da TPM é fundamentalmente clínico e baseado na anamnese e no exame físico geral e especial. Na TPM, o quadro clínico deve repetir-se pelo menos por três ciclos consecutivos. Assim, é importante uma avaliação prospectiva para caracterizá-lo. O principal objetivo do exame clínico é o diagnóstico e a exclusão das doenças orgânicas que possam mimetizar a TPM (FEBRASGO, 2010).

Um conjunto específico de sintomas durante a TPM interfere no relacionamento interpessoal, o que pode gerar uma alteração temporária nas relações e contatos sociais, aumento da ocorrência de acidentes, e uma redução da produtividade nos estudos e/ou trabalho (AZEVEDO MRD et al., 2006). Mediante isto, o presente estudo teve por objetivo verificar os sintomas e consequências da TPM mais ocorrentes em acadêmicas de uma Universidade do Sul de Minas Gerais, Brasil, e sua influência em suas rotinas e nas atividades universitárias, permitindo ampliar a visão e a abordagem dessa síndrome.

\section{MÉTODOS}

Trata-se de um levantamento descritivo, do tipo transversal, com abordagem quantitativa, desenvolvido por pesquisadores devidamente treinados, calibrados e capacitados para a aplicação uniforme do questionário. A amostra foi composta por estudantes do sexo feminino, com faixa etária entre os 18 e 45 anos, regularmente matriculadas do $1^{\circ}$ (primeiro) ao $3^{\circ}$ (terceiro) ano de cursos de graduação 
(Administração, Agronomia, Biomedicina, Ciências Contábeis, Direito, Educação Física, Enfermagem, Engenharia Civil, Farmácia, Medicina, Medicina Veterinária, Nutrição, Odontologia e Psicologia), na Universidade José do Rosário Vellano (Unifenas-Alfenas), entre Fevereiro e Março de 2017.

Este estudo foi aprovado pelo Comitê de Ética em Pesquisa da UNIFENAS tendo parecer favorável sob no 1.731.607. As participantes do estudo emitiram concordância por meio do Termo de Consentimento Livre e Esclarecido (TCLE), conforme a Resolução 466/12 (CNS, 2012), após terem sido informadas sobre os objetivos do estudo, riscos, benefícios, garantia do anonimato e do direito de desistência em qualquer fase da mesma.

A escolha de um grupo constituído por universitárias jovens foi fundamentada na justificativa do grau de escolaridade. Levou-se em consideração também o fato da TPM poder impactar diretamente no desempenho acadêmico das alunas. Além disso, o fato de avaliarmos universitárias de diferentes cursos serve para comparação, uma vez que as tarefas de cada curso são distintas, de carga horária diferente e de períodos de estudo também variados. Assim, foi realizada uma amostragem aleatória estratificada para selecionar uma amostra representativa da população alvo. O número total de universitárias foi determinado considerando-se a proporção para qualquer sintoma $50 \%$, sendo, então, conservador, e para o nível de significância de $1 \%$, que o erro cometido não fosse maior ou menor que $0,2 \%$, conforme Hogg $\mathrm{RV}$ et al. (2005). Para que esta técnica de amostragem fosse aplicada foi necessário levantar informações a respeito do número de mulheres que estão cursando o primeiro ano e terceiro ano de cada curso da universidade. Sendo assim, o número de mulheres selecionado de cada curso foi proporcional ao número de mulheres que pertencem aos cursos, separadamente, nos anos estudados.

Para a coleta de dados foi elaborado um questionário que constou de 2 partes: a primeira para caracterização da população estudada composta por dados sócio-demográficos, e a segunda composta por 17 itens estruturados e semiestruturados sobre a TPM. Este questionário foi adaptado de Muramatsu $\mathrm{CH}$ et al. (2001).

Os resultados foram reportados em valores proporcionais quanto ao total. Os valores de $p$ foram obtidos para cada grupo de sinais e sintomas visando estabelecer o nível de significância destes durante a TPM, na amostra. Os testes inferenciais para proporção foram realizados considerando o teorema central do limite, pois o tamanho da amostra foi elevado e a proposição é assintótica. Primeiramente, foram realizados testes inferenciais unilaterais para verificar se as proporções dos sintomas eram estatisticamente superiores a 0 , ao nível de significância de 5\%, pelo teste informado por Hogg RV et al. (2005), que segue assintoticamente uma distribuição normal padrão. Os testes bilaterais para verificação de diferença estatística ao nível alfa de $5 \%$ entre duas proporções $p 1$ e p2 de tamanho amostral n1 e n2, cuja estatística segue assintoticamente um normal padrão, conforme Hogg RV et al. (2005). Esse teste foi utilizado para a verificação de diferença entre proporções. Para verificar uma estimativa do erro tipo II ( $\beta$ ), onde se assumiu para todo teste ser no máximo $5 \%$, foi utilizado o pacote do software $R$ "pwr" de Champely $S$ et al. (2018), onde esse erro foi calculado por simulações computacionais para os diferentes níveis de significância, proporções e tamanhos amostrais de forma semelhante à sugerida por Cohen J (1988).

\section{RESULTADOS}

Este estudo foi realizado com 404 universitárias, sendo $213(52,8 \%)$ da Medicina, $50(12,4 \%)$ da Odontologia, $24(5,9 \%)$ do Direito, $24(5,9 \%)$ da Educação Física, $16(3,9 \%)$ da Medicina Veterinária, 14 (3,4\%) da Psicologia, 14 (3,4\%) da Biomedicina, 11 (2,7\%) da Nutrição, 11 (2,7\%) da Enfermagem, 9 (2,2\%) da Engenharia Civil, 5 (1,2\%) da Farmácia, 5 (1,2\%) da Agronomia, 4 (0,9\%) das Ciências Contábeis e 4 $(0,9 \%)$ da Administração. Na Tabela 1 estão sumarizadas as informações socioeconômicas das estudantes entrevistadas. Entre as 404 universitárias, observou-se que a faixa etária mais frequente foi 18-24 anos, correspondendo a $87 \%$ da amostra $(n=352) ; 9 \%$ de $25-30$ anos $(n=38) ; 3 \%$ de $30-40$ anos $(n=12)$; e apenas $1 \%$ acima dos 40 anos $(n=2)$. Quanto ao estado civil, $94 \%$ são solteiras $(n=382), 5 \%$ casadas $(n=18), 0,5 \%$ divorciadas $(n=2)$ e $0,5 \%$ viúvas $(n=2)$. Quando questionadas sobre suas atividades sexuais, $78 \%$ das entrevistadas referiram-se como praticantes e $22 \%$ não praticantes. 
Tabela 1 - Perfil socioeconômico das estudantes entrevistadas.

\begin{tabular}{|c|c|c|c|c|c|}
\hline Variável & $\mathbf{N}$ & $\%$ & Variável & $\mathbf{N}$ & $\%$ \\
\hline Curso & & & Idade & & \\
\hline Administração & 4 & 0,9 & 18 a 24 & 352 & 87 \\
\hline Agronomia & 5 & 1,2 & 25 a 30 & 38 & 9 \\
\hline Biomedicina & 14 & 3,4 & 30 a 40 & 12 & 3 \\
\hline Ciências Contábeis & 4 & 0,9 & Acima de 40 & 2 & 1 \\
\hline Direito & 24 & 5,9 & & & \\
\hline Educação Física & 24 & 5,9 & Estado civil & & \\
\hline Enfermagem & 11 & 2,7 & Solteira & 382 & 94 \\
\hline Engenharia Civil & 9 & 2,2 & Casada & 18 & 5 \\
\hline Farmácia & 5 & 1,2 & Divorciada & 2 & 0,5 \\
\hline Medicina & 213 & 52,8 & Viúva & 2 & 0,5 \\
\hline Medicina Veterinária & 16 & 3,9 & & & \\
\hline Nutrição & 11 & 2,7 & Atividade sexual & & \\
\hline Odontologia & 50 & 12,4 & Praticante & 315 & 78 \\
\hline Psicologia & 14 & 3,4 & Não praticante & 89 & 22 \\
\hline Total & 404 & 100 & $\bar{T}$ Total & 404 & 100 \\
\hline
\end{tabular}

Fonte: Próprios autores, 2019.

Nas Tabelas 2 a $\mathbf{5}$ podem ser observados que, com exceção de "temores" (sintomas físicos) e "visita-os mais" (relacionamento com a família), todas as proporções são significativas $(p<0,05)$. Todas as entrevistadas apresentavam algum sinal ou sintoma durante a TPM. Ainda nas Tabelas de 2 a 5, as proporções estão apresentadas para as variáveis em ordem crescente em relação à proporção observada. Verificou-se, par a par, quais variáveis são estatisticamente diferentes às demais.

Ao analisar as proporções de sintomas físicos apresentados pelas participantes na semana anterior ao início da menstruação, na Tabela 2, verifica-se que houve um grupo de maior frequência, constituído pelos sintomas: mamas inchadas e doloridas, desejo por comida, fome, dor de cabeça, inchaço em alguma parte do corpo, sensação de ganho de peso, aumento do desejo sexual, dor nas costas e sensação de peso no abdome.

Também se observou que houve um grupo intermediário com os sintomas: aumento do corrimento vaginal, aumento do apetite, diarreia e inchaço em todo corpo. Enquanto que no grupo identificado como sintomas de menor ocorrência houve a seguinte composição: ganho de peso real, dor nos músculos, náusea, constipação, dor nas juntas, diminuição do desejo sexual, sede, perda de apetite, tremores e outros. 
Tabela 2 - Proporções e valores-p para as proporções de "sintomas físicos" apresentados na semana anterior à menstruação pelas universitárias.

\begin{tabular}{|c|c|c|c|}
\hline Variável & $\mathrm{n}$ & $\%$ & Valor-p* \\
\hline \multicolumn{4}{|l|}{ Sintomas físicos } \\
\hline Mamas inchadas e doloridas & 248 & 61,4 & $<0,0001$ \\
\hline Desejo por alimentos & 242 & 59,9 & $<0,0001$ \\
\hline Fome & 220 & 54,4 & $<0,0001$ \\
\hline Dor de cabeça & 184 & 45,5 & $<0,0001$ \\
\hline \multicolumn{4}{|l|}{ Inchaço em alguma parte do } \\
\hline corpo & 154 & 38,1 & $<0,0001$ \\
\hline Sensação de ganho de peso & 139 & 34,4 & $<0,0001$ \\
\hline Aumento do desejo sexual & 129 & 31,9 & $<0,0001$ \\
\hline Dor nas costas & 114 & 28,2 & $<0,0001$ \\
\hline Sensação de peso no abdome & 102 & 25,2 & $<0,0001$ \\
\hline Aumento do corrimento vaginal & 91 & 22,5 & $<0,0001$ \\
\hline Aumento do apetite & 90 & 22,3 & $<0,0001$ \\
\hline Diarreia & 78 & 19,3 & $<0,0001$ \\
\hline Inchaço em todo o corpo & 72 & 17,8 & $<0,0001$ \\
\hline Ganho de peso real & 60 & 14,8 & $<0,0001$ \\
\hline Dor nos músculos & 50 & 12,3 & $<0,0001$ \\
\hline Náusea & 46 & 11,3 & $<0,0001$ \\
\hline Constipação & 35 & 8,6 & $<0,0001$ \\
\hline Dor nas juntas & 35 & 8,6 & $<0,0001$ \\
\hline Outros sintomas & 33 & 0,8 & $<0,0001$ \\
\hline Diminuição do desejo sexual & 22 & 5,5 & $<0,0001$ \\
\hline Sede & 18 & 4,4 & $<0,0001$ \\
\hline Perda de apetite & 15 & 3,7 & $<0,0001$ \\
\hline Tremores & 9 & 2,2 & 0,0012 \\
\hline
\end{tabular}

Fonte: Próprios autores, 2019.

*Significativo na amostra em questão. 
Em se tratando do estado emocional, pela Tabela 3, foi possível observar que o grupo de sintomas mais frequentes foi composto por: irritabilidade, seguido de impaciência, vontade de chorar, ansiedade e tristeza. O grupo identificado de menor incidência foi composto por: labilidade de humor, raiva, angústia, dificuldade de concentração, aumento do sono, tensão aumentada, desânimo e autodesvalorização, inquietação, interesse diminuído pelo estudo, distração, autopiedade e insônia.

Tabela 3 - Proporções e valores-p para as proporções de "sintomas psicológicos" apresentados na semana anterior à menstruação pelas universitárias.

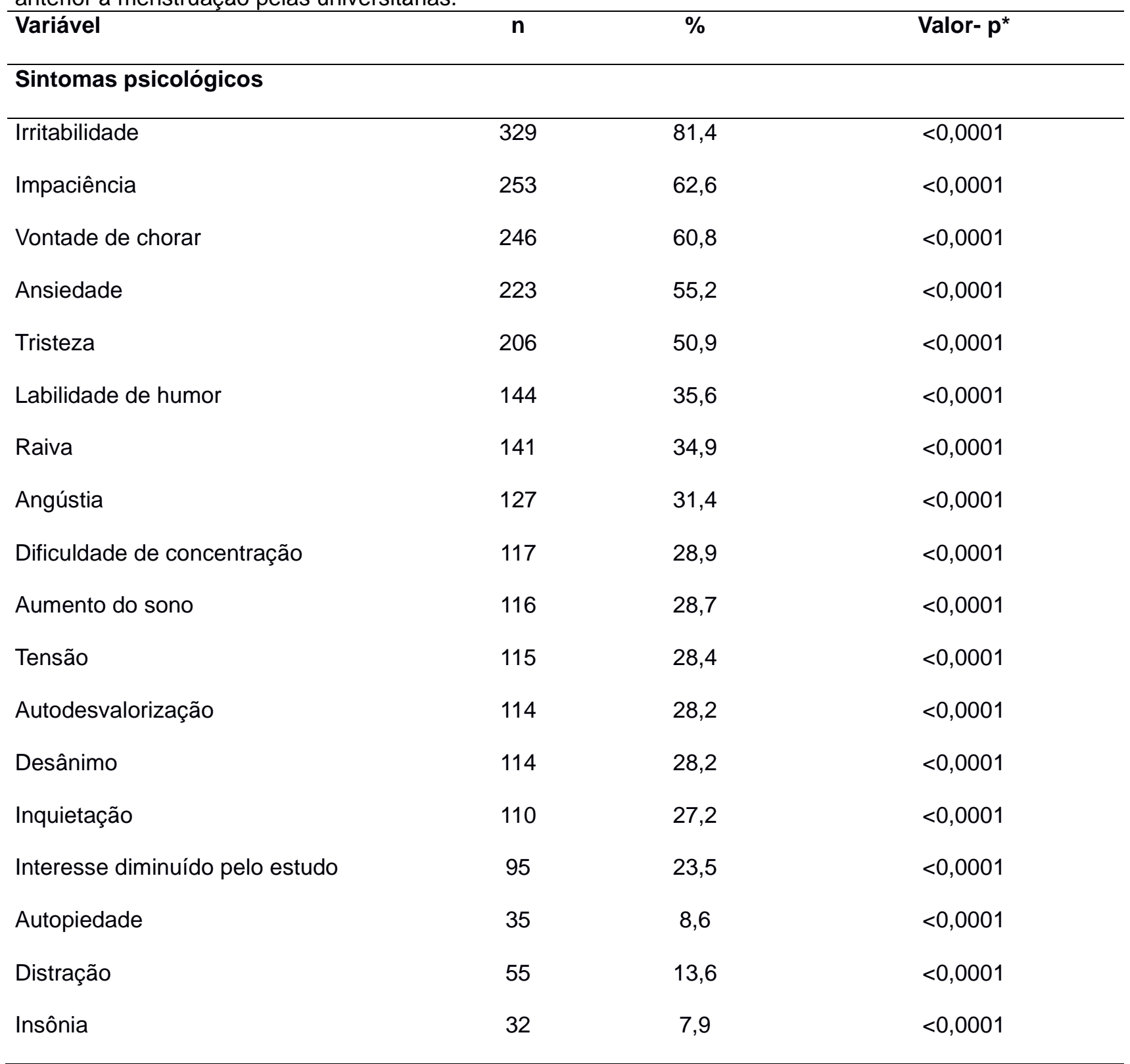

Fonte: Próprios autores, 2019. *Significativo na amostra em questão.

$\mathrm{Na}$ Tabela 4, quanto aos grupos de sintomas e consequências apresentados no trabalho e/ou na faculdade, a maior proporção de alunas reportou que respondem de forma hostil às pessoas, afrontam mais as pessoas, que ficam mais quietas e geram clima de tensão. Para esses fatores, o grupo identificado de menor frequência foi composto por alunas que: não conseguem terminar suas tarefas como de costume, faltam ou faltam mais de aulas, as que cometem "mais erros no trabalho ou faculdade", "acidentam-se mais facilmente", "tiram notas mais baixas nos trabalhos e provas", "chegam atrasadas", "levam mais bronca" e as que "perdem ou já perderam amizades". 
Tabela 4 - Proporções e valores-p para as proporções relacionadas à interferência da TPM nas atividades diárias apresentados na semana anterior à menstruação pelas universitárias.

\begin{tabular}{lccc}
\hline Variável & N & $\%$ & Valor-p $^{*}$ \\
\hline Consequências & & & \\
\hline Responde de forma hostil às pessoas & 215 & 53,2 & $<0,0001$ \\
Afronta mais as pessoas & 146 & 36,1 & $<0,0001$ \\
Quietude & 139 & 34,4 & $<0,0001$ \\
Gera clima de tensão & 109 & 27,0 & 0,0002 \\
Não consegue terminar suas tarefas como de costume & 86 & 21,3 & $<0,0001$ \\
Falta ou falta mais & 57 & 14,1 & $<0,0001$ \\
Comete mais erros no trabalho ou faculdade & 50 & 12,4 & $<0,0001$ \\
Acidenta-se mais facilmente & 43 & 10,6 & $<0,0001$ \\
Tira notas baixas nos trabalhos e provas & 40 & 9,9 & $<0,0001$ \\
Chega atrasada & 29 & 7,2 & $<0,0001$ \\
Leva mais bronca & 27 & 6,7 & $<0,0001$ \\
Perde amizades ou já perdeu & 16 & 3,9 & $<0,0001$ \\
\hline Fonte: Prón
\end{tabular}

Fonte: Próprios autores, 2019.

*Significativo na amostra em questão

Na Tabela 5 são identificados os grupos de fatores referentes ao relacionamento entre as entrevistadas e familiares. Identificaram-se como de maior ocorrência os fatores: briga mais com seus familiares, cria intrigas entre os familiares. Para o grupo de menor ocorrência houve a seguinte composição: outros tipos de comportamento como, ignoram os familiares, rompem relações mais facilmente e visitam mais frequentemente seus familiares.

Tabela 5 - Proporções e valores- $p$ para as relacionadas à interferência da TPM nos relacionamentos com familiares apresentados na semana anterior à menstruação pelas universitárias.

\begin{tabular}{lccc}
\hline Variável & $\mathbf{n}$ & $\%$ & Valor- $^{*}$ \\
\hline Relacionamento com familiares & & & \\
\hline Briga mais com familiares & 275 & 68,1 & $<0,0001$ \\
Cria intrigas com e entre eles & 174 & 43,1 & $<0,0001$ \\
Outros comportamentos & 79 & 19,5 & $<0,0001$ \\
Ignora-os & 77 & 19,0 & $<0,0001$ \\
Rompe relações facilmente & 44 & 10,9 & $<0,0001$ \\
Visita-os mais & 12 & 3,0 & 0,0002
\end{tabular}

Fonte: Próprios autores, 2019.

*Significativo na amostra em questão

\section{DISCUSSÃO}

Este estudo avaliou sintomas e alterações relacionadas à TPM em universitárias de uma instituição privada localizada no Sul de Minas Gerais. Aproximadamente $96 \%$ das entrevistadas encontrava-se com idade entre 18-30 anos, que é a faixa etária em que os sintomas e alterações da TPM mais se expressam, segundo da Silva CML et al. (2006). De acordo com da Silva CML et al. (2006), essa taxa foi verificada entre $62 \%$ e $66 \%$, o que condiz parcialmente com os resultados desse estudo, que com exceção do sintoma psicológico "irritabilidade", que apresentou ocorrência de aproximadamente $81 \%$, os demais fatores que mais se apresentaram no presente estudo estavam próximos a essa taxa. 
Após o início da menarca, as jovens referem no período antecedente à menstruação: alterações física, psíquica ou comportamental, geralmente, de pequena intensidade. Portanto, tais alterações não interferem significativamente no dia-a-dia, inclusive, algumas vezes, podem apresentar-se como inerentes a Síndrome da Adolescência Normal (SAN). Contudo, atualmente, cada vez mais mulheres apresentam sintomatologia intensa, levando a alterações significativas relativas aos compromissos sociais e tarefas do cotidiano, causando distúrbios no relacionamento familiar e social, e dificultando o desempenho nos estudos e/ou no âmbito profissional (APPROBATO MS et al., 2001).

Quando verificados os sintomas e consequências dos grupos de maior incidência identificados nesse estudo com os sintomas e consequências de maior frequência absoluta nos estudos reportados na literatura, foi verificada uma grande aproximação dos resultados. No presente estudo, ainda em concordância ao trabalho apresentado por da Silva CML et al. (2006), mulheres que apresentam a TPM, apresentam um conjunto de sintomas físicos, emocionais e comportamentais, que, segundo os autores, ocorrem de forma cíclica no período fértil, e podem ser variáveis na quantidade e na intensidade. Esses sintomas se manifestam na semana anterior à menstruação, ou seja, na fase lútea tardia do ciclo menstrual e aliviam-se com o início do fluxo menstrual.

No estudo de Diniz MS et al. (2013), realizado no ambulatório de ginecologia do Hospital das Clinicas da Universidade de São Paulo, verificou-se que o principal sintoma identificado durante o período da TPM foi à irritabilidade, sendo que a proporção verificada, $86 \%$, foi próxima ao do presente estudo, $81 \%$. Os sintomas identificados nos grupos de maior ocorrência no presente estudo e que coincidiram com os sintomas mais frequentes encontrados por Costa YR et al. (2007), foram: mudança de humor, tristeza, irritabilidade, ansiedade e aumento do apetite.

Em comparação ao estudo reportado por Luz JM et al. (2015), realizado em uma instituição pública de ensino superior no Piauí com acadêmicas do curso de enfermagem, os fatores relacionados com sintomas físicos e emocionais que coincidiram entre os mais frequentes com o presente estudo, foram: mamas inchadas, inchaço em outra parte do corpo e dor de cabeça. Os sintomas emocionais mais prevalentes foram: irritabilidade, impaciência, vontade de chorar e ansiedade. Quando comparados os índices de maior frequência nas consequências ocasionadas pela TPM na vida das acadêmicas, houve congruência com a literatura referente aos fatores: dar respostas hostis às pessoas e gerar clima tenso. Para as variáveis relacionadas com o relacionamento familiar ou com o parceiro no período da síndrome, os distúrbios emocionais mais frequentes em ambos os estudos, foram as "brigas".

Em comparação com o estudo realizado por Muramatsu CH et al. (2001), os sintomas físicos mais relatados e que estão presentes no grupo de maior frequência do presente estudo foram: mamas inchadas e dor de cabeça, enquanto que os emocionais foram: impaciência, irritabilidade e vontade de chorar. Como no estudo de Muramatsu $\mathrm{CH}$ et al. (2001), pode-se observar no presente estudo que as consequências mais ocorrentes da síndrome na vida e no cotidiano das entrevistadas foram: quietude, responder de forma mais hostis às pessoas e influenciar nas relações interpessoais. A consequência "brigar mais com seus familiares" foi outro fator também verificado por Muramatsu CH et al. (2001) e no presente estudo.

Diferente do reportado por Diniz MS et al. (2013), o presente estudo não reporta o fator "perda de apetite" como verificado no grupo sintomas físicos com alta ocorrência, sendo que esse fator se apresentou em apenas $3,71 \%$ das entrevistadas (estatisticamente não significante, mesmo sendo superior a zero ao nível de significância adotado). Por outro lado, o aumento do apetite, foi identificado no grupo de proporção observada intermediária, com uma proporção de observações igual a 22,28\%.

Alimentos ricos em carboidratos, como o chocolate, são ricos em triptofano que, quando metabolizado, se transforma em serotonina. Tal substância proporciona a sensação de bem-estar e melhora dos sintomas, como irritabilidade, depressão, tensão e ansiedade, que são vistos durante a TPM. Portanto, o desejo por alimentos específicos, observado em $59,9 \%$ das entrevistadas, seria uma forma inconsciente de melhorar seu estado disfórico, aumentando os níveis de serotonina e resgatando o equilíbrio, como forma de alívio (DINIZ MS et al., 2013). 


\section{CONCLUSÃO}

No presente estudo concluímos que todas as estudantes entrevistadas apresentaram algum tipo de sinal e sintoma, físicos e psicológicos, durante o período de TPM, e tais alterações de comportamento geram consequências em ambientes externos (fora de casa) e familiar. Os resultados encontrados apresentam uma adicional fonte de informações relacionada ao tema "TPM" e os mais frequentes sintomas e consequências relacionadas a esta condição, visando um entendimento sobre grupos populacionais específicos (como o universitário) de forma mais ampla e como essa síndrome pode influenciar as atividades diárias de uma acadêmica.

\section{REFERÊNCIAS}

1. APPROBATO MS et al. Síndrome pré-menstrual e desempenho escolar. Rev Bras Ginecol Obstet, 2001; 23(7): 459-62.

2. AZEVEDO MRD et al. Síndrome pré-menstrual em adolescentes: um estudo transversal dos fatores biopsicossociais. Arquivos Médicos do ABC, 2006; 31(1): 12-17.

3. BRILHANTE AVM et al. Síndrome pré-menstrual e síndrome disfórica pré-menstrual: aspectos atuais. Femina, 2010; 38(7): 373-378.

4. CHAMPELY S et al. Package "pwr" from software R, 2018.

5. COHEN J. Statistical power analysis for the behavioral sciences. New York: Lawrence Erlbaum, 2 ed., 1988.

6. CONSELHO NACIONAL DE SAÚDE - CNS (BRASIL), resolution 466. Brasília, Dezember $12,2012$. http://www.conselho.saude.gov.br/web_comissoes/conep/index.html. Accessed June 3, 2018.

7. COSTA YR, FAGUNDES RLM, CARDOSO BR. Ciclo menstrual e consumo de alimentos. Rev Bras Nutr Clín, 2007; 22(3): 203-209.

8. DINIZ MS, et al. Prevalência da Síndrome Pré-menstrual e seus Principais Sintomas Observados em Acadêmicas do Curso de Medicina de uma Faculdade do Sul de Minas Gerais. Revista Ciências em Saúde, 2013; 3(2): 43-59.

9. FEDERAÇÃO BRASILEIRA DAS ASSOCIAÇÕES DE GINECOLOGIA E OBSTETRÍCIA (FEBRASGO). Manual de orientação em trato genital inferior e colposcopia: vulvovaginites. São Paulo: FEBRASGO, 2010.

10. HOGG RV, MCKEAN J, CRAIG AT. Introduction to mathematical statistics, New York: Pearson, 6 ed., 2005.

11. LUZ JM, et al. Consequências da Síndrome Pré-Menstrual em Acadêmicas de Enfermagem. ReTEP, 2015; 7(2): 1537-1541.

12. MURAMATSU $\mathrm{CH}$, et al. Consequências da síndrome da tensão pré-menstrual na vida da mulher. Rev Esc Enferm USP, 2001; 35(3): 205-213.

13. PAIVA PSC, PAULA LB, NASCIMENTO LLO. Tensão Pré-Menstrual (TPM): uma revisão baseada em evidências científicas. Femina, 2010; 38(6): 311-315.

14. SILVA CML, et al. Estudo populacional de síndrome pré-menstrual. Rev Saude Publica, 2006; 40(1): 47-56. 